\title{
Extremely metal-poor stars in dwarf galaxies
}

\author{
Anna Frebel ${ }^{1}$, Joshua D. Simon ${ }^{2}$, Evan Kirby ${ }^{3}$, \\ Marla Geha ${ }^{4}$, and Beth Willman ${ }^{5}$ \\ ${ }^{1}$ Harvard-Smithsonian Center for Astrophysics, Cambridge, MA 02138, USA \\ email: afrebel@cfa.harvard.edu \\ ${ }^{2}$ Observatories of the Carnegie Institution of Washington, \\ Pasadena, CA 91101, USA \\ email: jsimon@ociw.edu \\ ${ }^{3}$ California Institute of Technology, Pasadena, CA 91106, USA \\ email enk@astro.caltech.edu \\ ${ }^{4}$ Astronomy Department, Yale University, New Haven, CT 06520, USA \\ email marla.geha@yale.edu \\ ${ }^{5}$ Haverford College, Haverford, PA 19041, USA \\ email: bwillman@haverford.edu
}

\begin{abstract}
We present Keck/HIRES spectra of six metal-poor stars in two of the ultra-faint dwarf galaxies orbiting the Milky Way, Ursa Major II and Coma Berenices, and a Magellan/MIKE spectrum of a star in the classical dwarf spheroidal galaxy (dSph) Sculptor. Our data include the first high-resolution spectroscopic observations of extremely metal-poor stars $([\mathrm{Fe} / \mathrm{H}]<-3.0)$ not belonging to the Milky Way $(\mathrm{MW})$ stellar halo field population. We obtain abundance measurements and upper limits for up to 26 elements between carbon and europium. The stars span a range of $-3.8<[\mathrm{Fe} / \mathrm{H}]<-2.3$, with the ultra-faints having large spreads in Fe. A comparison with MW halo stars of similar metallicity reveals substantial agreement between the abundance patterns of the ultra-faint dwarf galaxies and Sculptor and the MW halo for the light, $\alpha$ and iron-peak elements (C to $\mathrm{Zn}$ ). This agreement contrasts with the results of earlier studies of more metal-rich stars $(-2.5 \lesssim[\mathrm{Fe} / \mathrm{H}] \lesssim-1.0)$ in more luminous dwarfs, which found significant abundance discrepancies with respect to the MW halo data. The abundances of neutron-capture elements ( $\mathrm{Sr}$ to $\mathrm{Eu}$ ) in all three galaxies are extremely low, consistent with the most metal-poor halo stars, but not with the typical halo abundance pattern at $[\mathrm{Fe} / \mathrm{H}] \gtrsim-3.0$. Our results are broadly consistent with a galaxy formation model which predicts that massive dwarf galaxies are the source of the metal-rich component $([\mathrm{Fe} / \mathrm{H}]>-2.5)$ of the $\mathrm{MW}$ inner halo, but we propose that dwarf galaxies similar to the dSphs are the primary contributors to the metal-poor end of the metallicity distribution of the MW outer halo.
\end{abstract}

Keywords. stars: abundances, stars: Population II, Galaxy: formation, Galaxy: halo, galaxies: dwarf, (galaxies:) Local Group, galaxies: stellar content, (cosmology:) early universe

\section{Introduction}

According to prevailing theories of the early Universe, the recombination of the plasma of electrons and light nuclei (mostly $\mathrm{H}$ and $\mathrm{He}$, with trace amounts of $\mathrm{Li}$ ) emerging from the Big Bang was followed by the Dark Ages, during which space was filled with gas and radiation but no sources of light. This era ended with the formation and ignition of the very first (Pop III) stars. These presumably very massive $\left(\sim 100 \mathrm{M}_{\odot} ;\right.$ Bromm \& Larson 2004) objects soon died as energetic supernovae. The freshly synthesized metals expelled by those explosions provided the first effective coolants for the interstellar medium. This pollution set the stage for the second generation of stars (Pop II), which included stars over a wide range of masses. The low-mass $\left(\mathrm{M} \leqslant 0.8 \mathrm{M}_{\odot}\right)$ early Pop II stars, which 
formed from gas enriched by the first stars, live longer than a Hubble time and are thus still observable. In their atmospheres, these stars preserve the characteristic chmemical signature of the gas at the time of their birth - prior to the formation of the Galaxy.

Metal-poor stars represent the local equivalent of the high-redshift Universe because they supply us with direct information on conditions shortly after the Dark Ages ("stellar archaeology"). We are thus provided with a unique window on early star formation and the onset of galaxy formation. Detailed studies of these oldest stars can therefore address a broad range of astrophysical issues, including the Pop III initial mass function, the formation of the first low-mass stars, the yields of the first supernovae and relevant nucleosynthesis processes and sites, the relation between chemistry and kinematics, and whether the Milky Way's stellar halo was built from accreted satellites.

\section{High-resolution spectroscopy of stars in dwarf galaxies}

While there has been intense interest recently in the most metal-poor Galactic stars (e.g., Frebel et al. 2005), progress in discovering new stars with $[\mathrm{Fe} / \mathrm{H}] \lesssim-4$ has stalled as the best halo candidates from the Hamburg/ESO survey and the SDSS have been exhausted. The next frontier in the search for the most ancient stars lies in the nearby dwarf satellites of the Galaxy (e.g., ). Earlier studies suggested that dwarf galaxies did not contain any stars below $[\mathrm{Fe} / \mathrm{H}]=-3$, sparking a debate about the viability of the Searle \& Zinn (1978) paradigm (Helmi et al. 2006). We have shown that this claim stems merely from biases in the search technique (Kirby et al. 2008; Cohen \& Huang 2009). With improved methods for identifying the lowest-metallicity objects, the sample of extremely metal-poor $(\mathrm{EMP} ;[\mathrm{Fe} / \mathrm{H}]<-3)$ stars in dwarf galaxies is primed to explode. We here present the first results of an ongoing effort to observe the brightest and most metal-poor stars in dwarf galaxies with high-resolution spectroscopy.

The metallicity-luminosity relationship of dwarf galaxies (Kirby et al. 2008) indicates that the lowest luminosity dwarfs should contain the most metal-poor stars. Of the 15 newly identified EMP stars in the ultra-faint dwarfs (Kirby et al. 2008), we observed six with high-resolution spectroscopy. Three are located in each of Ursa Major II (UMa II) and Coma Berenices (ComBer). Two of the stars in UMa II are extremely metal-poor having metallicities of $[\mathrm{Fe} / \mathrm{H}]<-3.0$. We recently also discovered the first extremely metal-poor star in a classical dwarf galaxy from a sample of 380 stars located in the Sculptor dSph (Kirby et al. 2009). The metallicity of $[\mathrm{Fe} / \mathrm{H}]=-3.8$ was confirmed from the high-resolution spectrum taken with Magellan/MIKE (Frebel et al. 2010b). This remarkable finding suggests that more such low-metallicity stars could soon be identified in these more luminous systems.

Further details on the observations and analysis techniques are given in Frebel et al. (2010a). We compare these new observations with previous abundance studies of stars in the Milky Way halo and the brighter, classical dSphs (see Fig. 1). The ultra-faint dwarf galaxies have large internal metallicity spreads. Confirming the earlier results at lower spectral resolution of Simon \& Geha (2007), Kirby et al. (2008), and Norris et al. (2008), we find that our three stars in each galaxy span a range of $\sim 0.9 \mathrm{dex}$ in $[\mathrm{Fe} / \mathrm{H}]$ in UMa II, $\sim 0.6$ dex in ComBer, and more than 2 dex in Sculptor. Such spreads indicate early star formation in multiple proto-dwarf galaxies that later merged, extended star formation histories, or incomplete mixing in the early ISM.

We provide the first evidence that the abundance patterns of light elements $(Z<30)$ in the ultra-faint dwarfs as well as in Sculptor at $[\mathrm{Fe} / \mathrm{H}] \sim-3.8$ are remarkably similar to the Milky Way halo. This is in contrast to what is seen in the brighter dSphs (e.g. Venn et al. 2004) at higher metallicites. The agreement we find suggests that the metal-poor end of the MW halo population could have been built up from destroyed dwarf galaxies. 
Neutron-capture elements are of extremely low abundances in the ultra-faint dwarf galaxies, as well as in Sculptor. Particularly in Sculptor, but also in ComBer, the Ba and Sr values observed are well below the abundances found in typical MW halo stars with similar Fe abundances (Frebel et al. 2010b). The low neutron-capture abundances may represent a signature typical of stars with $[\mathrm{Fe} / \mathrm{H}] \lesssim-2.0$ in dwarf galaxies. Similarly low values have been found in Hercules (Koch et al. 2008) and Draco (Fulbright et al. 2004).

The results above are broadly consistent with the predictions of currently favored cosmological models (e.g. Robertson et al. 2005, Johnston et al. 2008). The majority of the mass presumably in the inner part of the stellar halo (at $[\mathrm{Fe} / \mathrm{H}] \sim-1.2$ to -1.6 ) was formed in much larger systems such as the Magellanic Clouds. Our results now support a scenario where the ultra-faint dwarf galaxies contributed some individual metal-poor
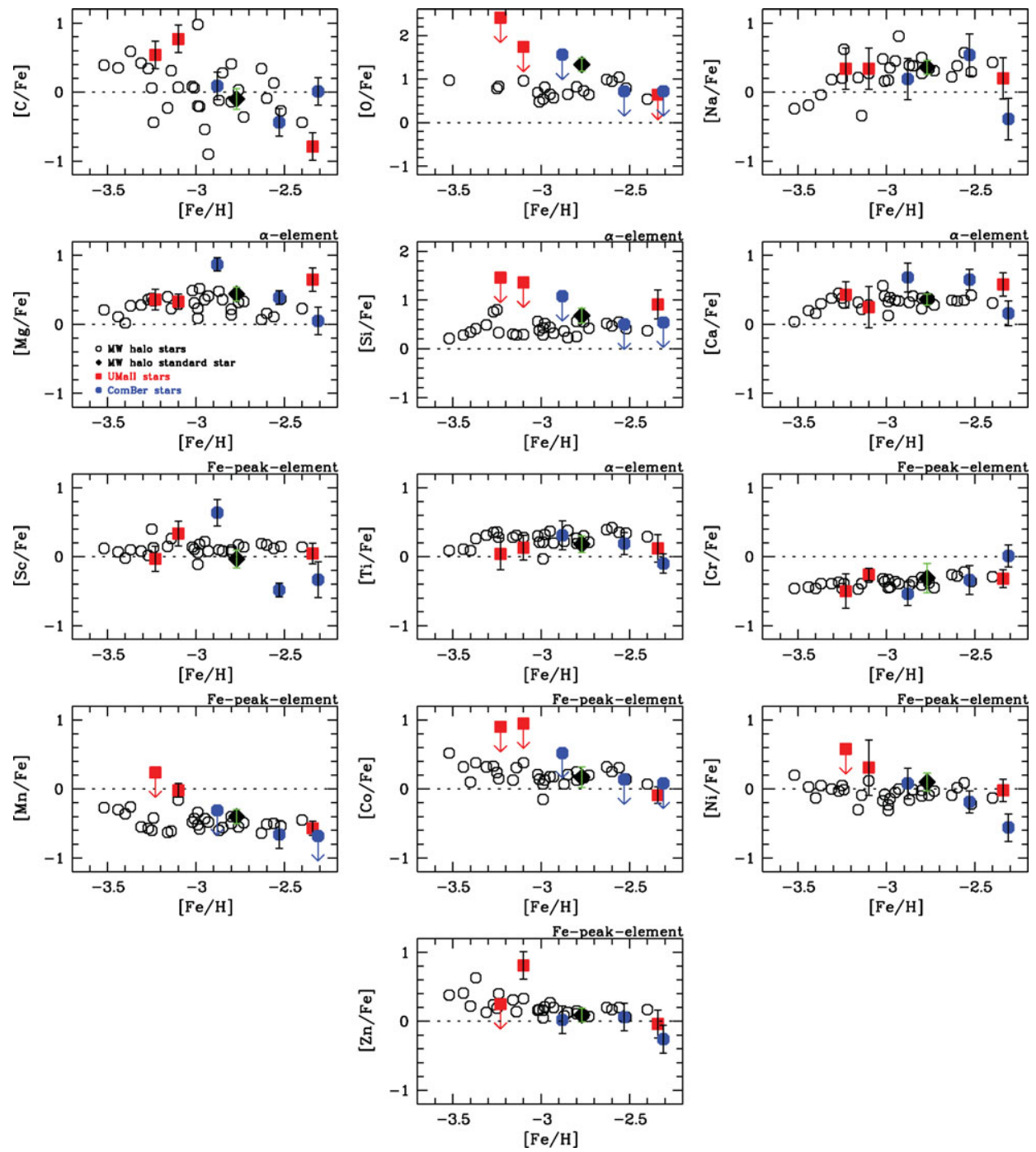

Figure 1. Abundance ratios $([\mathrm{X} / \mathrm{Fe}])$ as a function of metallicity $([\mathrm{Fe} / \mathrm{H}])$ for light and iron-peak elements in comparison with those of Cayrel et al. (2004). Red squares indicate UMa II stars, blue circles show ComBer stars, open black circles are the Cayrel et al. halo sample. The halo star HD122563 is shown by a black diamond. (Taken from Frebel et al. (2010a).) 
stars that are now found primarily in the outer Galactic halo (although not exclusively). However, these systems may not have been suffciently numerous to acount for the entire metal-poor end of the Fe metallicity distribution of the Milky Way halo. Since the classical dSphs contain more stellar mass and have been shown to also contain at least some of the most metal-poor stars, they could have been a major source of the lowest-metallicity halo content.

\section{Conclusions}

It appears that the hunt for the most metal-poor stars may have just begun since these dwarfs host a large fraction of low-metallicity stars, perhaps even much higher than what has so far been inferred for the Galactic halo (Schörck et al. 2009). The currently available stars already provide important clues on early star and galaxy formation, the nature and sites of the first stars, the onset of chemical enrichment and the relevant nucleosynthesis processes. The detailed abundance patterns of the stars in UMa II, ComBer and Sculptor are strikingly similar to that of the Milky Way stellar halo, thus renewing support for dwarf galaxies as the building blocks of the halo. Future discoveries of additional faint dwarf galaxies (for example with Skymapper) will enable the identification of many more metal-poor stars in systems similar to UMa II and ComBer. But also the brighter dSphs have to be revisited for their metal-poor content (Kirby et al. 2009). More stars at the lowest metallicities are clearly desired to better quantify the emerging chemical signatures and to solidify our understanding of the early Galaxy assembly process. Only in this way can the hierarchical merging paradigm for the formation of the Milky Way be put on firm observational ground. The next-generation optical telescopes will facilitate a great advance in this field since the vast majority of the stars in any dwarf galaxy are too faint to be followed-up with high-resolution spectroscopy on current 6-10 m telescopes. These observations, however, are mandatory for investigating how the surviving dwarfs are linked to their early counterparts that built the halo.

\section{References}

Bromm, V. \& Larson, R. B. 2004, ARAA, 42, 79

Cayrel, R. et al. 2004, A\&SA, 416, 1117

Cohen, J. G. \& Huang, W. 2009, ApJ, 701, 1053

Frebel, A. et al. 2005, Nature, 434, 871

Frebel, A., Simon, J. D., Geha, M., \& Willman, B. 2010a, ApJ, 708, 560

Frebel, A., Kirby, E., \& Simon, J. D. 2010b, Nature in press, astro-ph/0912.4734

Fulbright, J. P., Rich, R. M., \& Castro, S. 2004, ApJ, 612, 447

Helmi, A. et al. 2006, ApJ,651, L121

Johnston, K. V. et al. 2008, ApJ, 689, 936

Kirby, E. N. et al. 2009, ApJ, 705, 328

Kirby, E. N., Simon, J. D., Geha, M., Guhathakurta, P., \& Frebel, A. 2008, ApJ, 685, L43

Koch, A., McWilliam, A., Grebel, E. K., Zucker, D. B., \& Belokurov, V. 2008, ApJ, 688, L13

Norris, J. E. et al. 2008, ApJ, 689, L113

Robertson, B., Bullock, J. S., Font, A. S., Johnston, K. V. \& Hernquist, L. 2005, ApJ, 632, 872

Schörck, T. et al. 2009, A\&AA, 507, 817

Searle, L. \& Zinn, R. 1978, ApJ, 225, 357

Simon, J. D. \& Geha, M. 2007, ApJ, 670, 313

Tolstoy, E., Hill, V., \& Tosi, M. 2009, ARA\&A, 47, 371 